\title{
Phase separation instabilities and magnetism in two dimensional square and hexagonal Hubbard model
}

\author{
A.N. Kocharian ${ }^{a} *$, Kun Fang ${ }^{b}$, G. W. Fernando ${ }^{b}$ and A. V. Balatsky

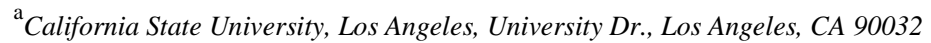 \\ ${ }^{\mathrm{b}}$ University of Connecticut, Storrs, CT 06269, USA \\ ${ }^{c}$ AlbaNova Univ. Center Nordita, SE-106 91, Stockholm, Sweden
}

Elsevier use only: Received date here; revised date here; accepted date here

\begin{abstract}
The variational cluster approximation is applied to rigorously calculate intrinsic local electron correlations in bipartite square and honeycomb Hubbard lattices. The Mott-Hubbard gap at half filling is manifested by a smooth metal-insulator transition in both lattices in agreement with the generic two-dimensional phase diagram. However, a density variation with the chemical potential shows the distinct structural differences away from half filling. The square lattice exhibits electron density discontinuity accompanied with spontaneous transition from antiferromagnetic Mott-Hubbard insulator into nonmagnetic metal. The spectral density anomaly and spin susceptibility peaks also are signaling on coexistence of hole rich metallic and hole poor insulating regions. In contrast, honeycomb lattice does not show density anomaly but displays a smooth transition with continuous evolution of homogenous metallic state. These calculations provide strong evidence for spontaneous phase separation instability found in our quantum cluster calculations at moderate $U$
\end{abstract}

(C) 2014 Elsevier B.V. All rights reserved

PACS: 72.25.-b, 73.40.Gk, 73.23.Ad, 73.40.Kp, 73.63.-b

Keywords: VCA, Mott metal-insulator transition, square lattice, honeycomb structure, phase separation, phase coexistence, pairing

\section{Introduction}

Strongly correlated electronic materials, found in several transition metal oxides, give rise to a broad range of different phenomena, leading to complex phase diagrams, phase separation (PS) instabilities and spatial inhomogeneities with (nanoscale) variations of electron charge and spin densities [12]. It is believed that spontaneous phase separation, electronic and magnetic inhomogeneities are essential intrinsic properties for understanding of charge and spin pairing instabilities in variety of transition oxides, high- $\mathrm{T}_{\mathrm{C}}$ cuprates and manganites. Theoretical investigations of strongly correlated materials are based on the Hubbard model with moderate and strong interactions which are performed by numerical calculations of Hubbard and $t$ - $J$ models [3-5]. The exact calculations in small Hubbard clusters around optimal doping display incipient PS, spatial spin/charge inhomogeneities driven by electron interaction and lattice structures [6-8]. This provides a media with effective attraction of holes or parallel spins that give rise to superconductivity and ferromagnetism. The level crossings in charge and spin excitation gaps found in the finite square lattices generated by (finite) 8 and 10-site Betts unit cells [9] further points to the existence of the two quantum critical points at

* Corresponding author. Tel.: +1-323-343-2100; fax: +1-323-3343-2497.

E-mail address: armen.kocharian@ calstatela.edu 
moderate and strong interactions responsible for electron and spin density instabilities in large thermodynamic systems. However, cluster calculations always are invariably tied to some uncertainties due to size and boundary effects and obtained results still remain controversial. In variational cluster approximation (VCA) the coexistence of global antiferromagnetic and superconducting orders is obtained by introducing fictitious Weiss fields [10]. Here we address the local microscopic mechanism of possible phase separation in the Hubbard model when doping at moderate $U$ generates a variation of electron density with coexistence of nonmagnetic hole rich and hole poor antiferromagnetic (AF) regions. In this report, the local mechanism of electronic phase separation and geometric effects are studied using the VCA method without any additional long-range input. Recent discoveries suggest that density phase separation under doping often occur in the vicinity of a Mott-Hubbard metal-insulator transition (MIT) [11-17]. In contrast to one-dimensional (1d) Hubbard model at half filling in both square and honeycomb lattices the MIT occurs at finite Coulomb interaction $U_{c}$. In general, this transition at a finite $U_{\mathrm{C}}$ value might be an intrinsic property of the $2 \mathrm{~d}$ lattice due to the energy competition between itinerant and localized characters of electron charge and spin degrees of freedom. Here we study possible phase separation in square and honeycomb lattices from half-filling to optimal doping. We find strong evidence of phase separation in the square lattice at moderate $U \sim U_{\mathrm{c}}$. However, we do not find such phase separation in the honeycomb lattice under variation of $U$ or doping. We compare oneparticle spectral functions and transverse spin susceptibility in the relevant doping region and identify possible geometry-related spectral density anomaly at phase separation instability.

\section{Theoretical Model}

\subsection{Model}

We consider the standard Hubbard model $\hat{H}$

$$
\hat{H}=\sum_{\left\langle r, r^{\prime}\right\rangle \sigma}\left(-t \hat{c}_{r \sigma}^{+} \hat{c}_{r^{\prime} \sigma}+H . c .\right)+U \sum_{r} \hat{n}_{r \uparrow} \hat{n}_{r \downarrow}
$$

where $\hat{c}_{r^{\prime} \sigma}$ is the annihilation operator for electrons at site $r$ with spin projection $\sigma$ and $U$ is the on-site screened Coulomb repulsion. Here $\left\langle r, r^{\prime}\right\rangle$ denotes summation over the nearest neighbors with lattice parameter $a=r^{\prime}-r$. The coupling parameter $t$ is the transfer integral between the nearest neighbors.

\subsection{Variational cluster approximation}

Here we use the VCA grand canonical method [18] to solve the problem of phase separation in the Hubbard model on a large-size lattice by diagonalization in real space. This is a quantum cluster [19] extension of Potthoff's self-energy functional approach [20-22]. The VCA with exact diagonalization does not encounter the sign problem of Monte Carlo method at low temperature or away from half filling. The VCA method decomposes the lattice (original system) with one-particle parameters $t$ and on-site interaction parameter $U$, into isolated clusters (reference system) with modified one-particle parameters $t^{\prime}$ and the same on-site interaction parameter $U$. The Hubbard model on the cluster level is exactly solvable by either exact diagonalization [7] or Lanczos method [23]. The exact eigenvalues and eigenvectors can lead to an accurate implementation of the correlation effects through the self-energy term $(\Sigma)$. The VCA method sufficiently accurate for calculation of short-range correlation. The self-energy in the reference system is used as an approximation for the original infinite system, while the coupling between clusters is treated by cluster perturbation theory [22]. The grand potential $\Omega$ of the original system can be written as a functional of the self-energy $\Sigma$ provided the system is not at a critical point associated with a phase transition [20]:

$$
\Omega_{t}[\Sigma]=\Omega_{t^{\prime}}[\Sigma]-\operatorname{Tr} \ln \left(G_{0}^{-1}-\Sigma\right)-\operatorname{Tr} \ln \left(G_{0}^{\prime-1}-\Sigma\right)
$$

where $G_{0}\left(G_{0}^{\prime}\right)$ and $\Omega_{t}\left(\Omega_{t^{\prime}}\right)$ are the non-interacting Green's function and the grand potential of the original system (reference system). A variational calculation can be built, based on the above equation, by varying $t^{\prime}$. It has been shown [20] that $\Omega[\Sigma]$ are stationary at the exact self-energy and its value is the exact grand potential of the system. Because the evaluation of the stationary points of the functional is performed in the restricted selfenergy space of the reference system, instead of the full self-energy space, the ground state found in VCA is approximate. The size of the reference 
system (i.e., the size of the isolated clusters) determines the dimensionality of the self-energy space affects the accuracy of the results. However, the improvement of the accuracy by enlarging cluster size does not significantly affect the results.

\section{Results}

\subsection{Half filling}

The appearance of an energy gap in the VCA at finite $U_{\mathrm{C}}$ around the Fermi level in the one-particle excitation spectra in square and honeycomb geometries at half filling provide strong support for a smooth MIT in generic 2d bipartite lattice structures. The critical value approached from both sides of $U_{\mathrm{C}}$ in square and honeycomb lattices is approximately equal to $U_{\mathrm{C}}=1.5$ and 2.5 respectively. In the VCA we do not include any long-range Weiss field in the reference system; therefore, the results of our calculations reflect intrinsic local properties of the $2 \mathrm{~d}$ Hubbard model, consistent with results in [24-26]. Fig. 1 shows the density of states in honeycomb lattice at various $U$. The MIT gap monotonously increases as $U$ ncreases beyond $U_{\mathrm{C}}$. In honeycomb lattice using VCA with fictitious AF field we find also a continuous transition from nonmagnetic into antiferromagnetic state at $U_{\mathrm{C}}=3$.

\subsection{Away from half-filling}

The density state The density of states in honeycomb lattice away from half-filling at $\omega=0$ in Fig. 1 (right) displays a stable metallic phase at all $U$ values. The local $\mathrm{AF}$ correlations in zero frequency transverse spin susceptibility gradually decrease with hole doping.

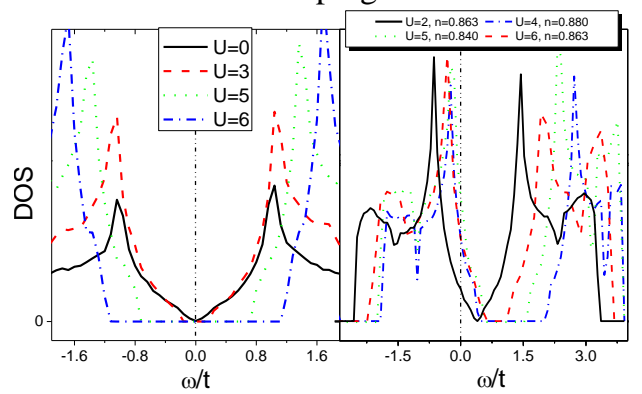

Fig. 1. The density of states in honeycomb lattice at different $U$ values. (left) At half-filling, zero frequency energy gap at $\omega=0$, opens at $U>U_{\mathrm{C}}$ and increases with $U$ monotonously. (right) Away half filling, the metallic $\mathrm{AF}$ state is a stable phase for all $U$.
However, the situation in square lattices is quite different and accompanied by phase separation instability.

\subsection{Phase separation}

The proximity to the MIT under doping has received renewed theoretical and experimental interest by attempts to understand the interplay of phase separation instabilities, spatial inhomogeneies and pairing, in copper-oxides and pnictides. Phase separation, caused by electron instabilities under doping, leads to the coexistence of inhomogeneous, hole-rich and hole-poor regions. This phenomenon closely resembles phase separation instabilities observed in the first order electronic phase transitions in rare earth materials [27]. The coexisting states with different electron densities $n$ share the same chemical potential $\mu$. The electron filling $n$ vs $\mu$ plots are obtained from our VCA calculations for the two different lattice structures at different Coulomb interactions $U$.

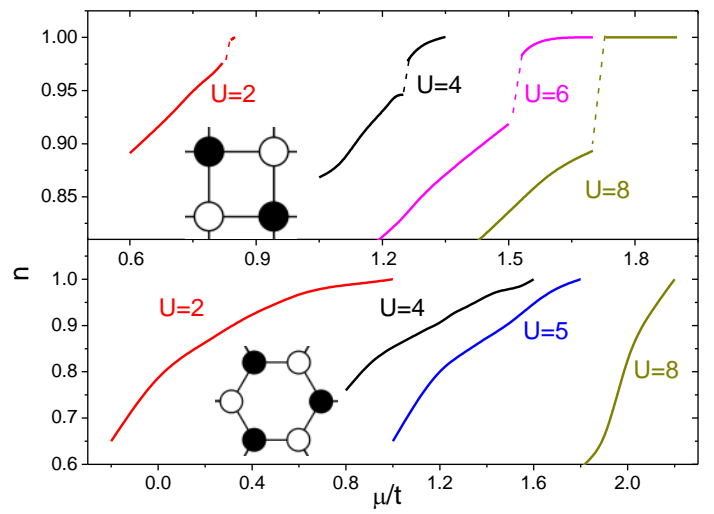

Fig. 2. The filling factor $n$ versus $\mu$ at different $U>U_{\mathrm{C}}$ values. (left) The discontinuities at distinct $n$ values in the square lattice with different $n$ at two edges of the step-like discontinuous transition have the same $\mu$ value, so the in underdoped region can coexists with hole rich metal. (right) The density in honeycomb lattice changes smoothly, without discontinuities, which implies the absence of phase separation. The corresponding reference systems used in the VCA calculation are shown in the insets

In Fig. 2, the plots with several $U$ values are shown at zero temperature. It is found that the plots for the square lattice show discontinuities where the dependence of density on $\mu$ is multivalued, and the electronic phase separation occurs in the square lattice at the vicinity of these discontinuities similar to the spontaneous phase separation at the first order phase transition. This implies that electron density 
is not homogeneous and experience inhomogeneous distribution in real space. For example, at $U=4$, the discontinuity of the plot appears at $\mu$ around 1.25 and the density changes abruptly from 0.946 to 0.977. The two states in equilibrium can coexist and have almost the same $\mu$. This means that the lattice at some critical electron concentration becomes phase separated which behaves as a mixture of two coexisting electron configurations with spatial (density) inhomogeneities. As $U$ increases, the discontinuity of the density also increases (see Fig. 1) which suggests that interaction plays an important role in the electronic phase separation. In addition, since no long-range order term (for spontaneous symmetry breaking) has been added in the variational calculation, so the ground state is driven purely by local electron correlations. Electronic phase separation in the square lattice is manifested at some finite critical $U_{\mathrm{C}}$, close to the MIT transition at half-filling. This result suggests that the nature of electronic phase separation in the square lattice is ultimately related to MIT, so the electronic phase separation is likely to be instigated by local Coulomb interaction. Similar results were also reported in using DCA [19]. In two dimensions phase separation requires a moderate Coulomb interaction so that it cannot be obtained at the weak coupling limit up to $U<U_{\mathrm{c}}$. The observed phase separation instabilities are signaled by a critical point $U_{\mathrm{C}}$ established by the onset of the first-order character of the transition by the proximity to the smooth MIT transition at zero doping [12-13]. In contrast, the density variation plots for the honeycomb lattice are smooth for all the $U$ values and there is no sign of electronic phase separation instabilities. Although the Mott-Hubbard transition at half-filling for the honeycomb lattice occurs at a larger $U>U_{\mathrm{c}}$, there are no indications of phase separation discontinuities in a similar region of hole doping. Since the honeycomb lattice and the square lattice are both bipartite structures with the same hopping and interaction parameters, this distinct behavior suggests that the strong interaction is not the only driving force for phase separation; the geometry or the symmetry of lattice is crucial and plays an equally important role. Combining results from our calculations, a phase diagram for the square lattice can be extracted as shown in Fig. 3 . The nonmagnetic metal (M), the Mott-Hubbard antiferromagnetic insulator (AFI) and phase separation (PS) are marked in the figure. This figure indicates that the square lattice has a phase separation region, in which the system is an inhomogeneous metal coexisting with AFI. The range of change in electron density at phase separation increases with $U$, which indicates the important role of electron correlations. There is also a transition from AFI into PS phase at large $U$ and small doping. This result in the VCA has been obtained without input of any additional order parameter. At given $U$ the doping with holes leads to transition from AFI into PS and then to metal. In general, increasing the pressure is equivalent to decreasing $U$. Thus, our phase diagram predicts the pressure induced transition also from AFI into PS phase and to metal phase. This phase diagram is consistent also with recently reported pressure and doping induced phase separations in cuprates [2830]. There are questions related to the size of the AF or metallic domains when the system is phase separated. The answer to such questions should be determined by the interaction strength and correlation length in the model [5]. These correlations are restricted to the cluster size in the VCA calculations, so it is not possible to address this question directly from our VCA calculation.

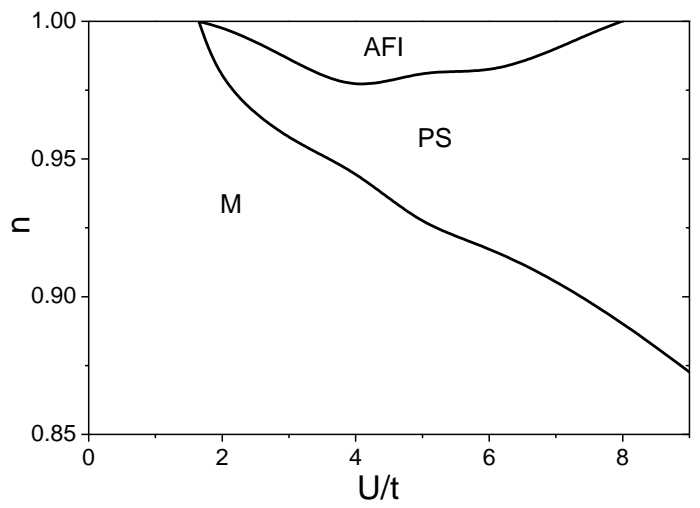

Fig. 3. Phase diagram of the Hubbard model in the square lattice The phase diagram is drawn in the space $U$ vs. hole concentration, $x=1-n$. The metallic phase exists when $U$ is relatively small or the doping level is high. Antiferromagnetic insulator is stable at moderate $U$ values and low doping. PS phase consists of mixed AFI and $\mathrm{M}$ states distributed inhomogeneously in the lattice.

\subsection{Spectral functions}

Using one-particle Green's function and the transverse spin susceptibility the VCA-based oneparticle excitation spectrum

$$
A(\vec{k}, \omega)=-2 \lim _{\eta \rightarrow+0}[\operatorname{Im} G(\vec{k}, \omega+i \eta)],
$$


is obtained in the momentum $k$ and frequency $\omega$ to study the folding/unfolding of the first Brillouin zone (FBZ) and zero frequency spin susceptibility $\kappa$ to measure a static local $\mathrm{AF}$ order at phase separation instability. We extracted the spectral function for the two densities right before and after the electronic phase separation. The single particle excitation spectrum at $U=4$ of a square lattice is shown in Fig. 4. By comparing the spectral functions at the two densities, one can find that a set of narrow spectra around the Fermi surface (FS) at $Q=( \pm \pi / 2 a, \pm \pi / 2 a)$ point (see the red arrow in Fig. 4 between the $\Gamma$ and $M$ points) which disappears when the density drops from 0.977 to 0.946 . This indicates that in momentum space the $Q$ point becomes unstable as phase separation occurs. The scattering transfer momentum $Q$ resides on the boundary of the folded (FBZ) due to the doubling of lattice parameter, since the primitive unit cell in AF state contains two sites. The FS coincides with the borders of AF FBZ with a complete "nesting" after translation by vector of AF local ordering Q. When the system undergoes a transition from the AF state into the normal state, the primitive unit cell of the square lattice changes from containing two sites to only one site; hence the FBZ is unfolded since the zone boundary does not go through the $Q$ point in the normal state.

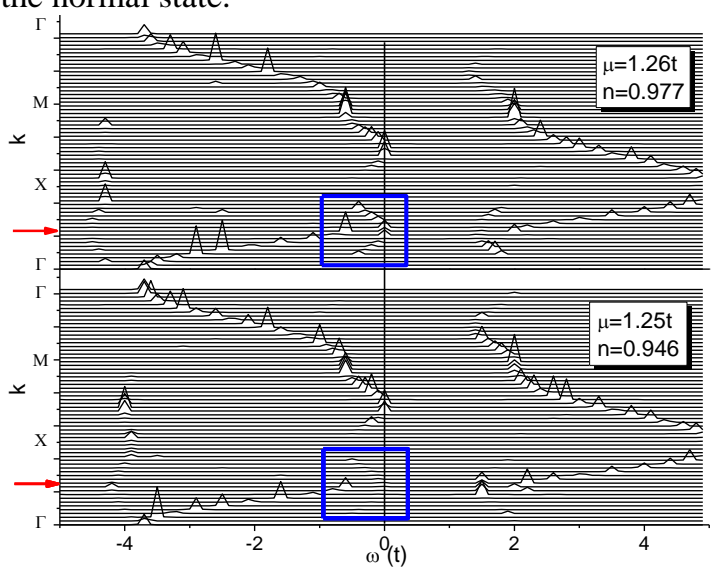

Fig. 4. One-particle excitation spectral functions for the Hubbard model at $U=4$ on square lattice right before and after phase separation are shown: (up) $\mu=1.26(n=0.977)$ is a Mott insulating phase with electron-hole symmetry and weak static AF order (small $K$ peak); (right) $\mu=1.25 \quad(n=0.947)$ is a normal metallic phase where the spin susceptibility peak disappears. The dotted line at $\omega=0$ denotes the Fermi level. At the higher electron density $(n=0.977)$, a part of spectra in (left), which is enclosed in the blue square, shows up around the $Q$ point (marked by red arrow) at the Fermi level, but it vanishes in (right) at the lower density $n=0.947$ (almost no peaks in the blue square).
The correlation between the disappearance of spectra and the reconstruction of FBZ boundaries (driven by doubling of lattice parameters) suggests that the Fermi surface instability is caused by the dramatic changes in the FBZ. Similar conclusions were drawn in [27-28] using the renormalization group (RG) method. In our calculation, similar Fermi surface instabilities are also seen in the square lattice with other interaction strengths as the system undergoes phase separation, so phase separation has a close connection to the Fermi surface instability. Our numerical studies in the square Hubbard lattice suggest that when the number of particles is slightly less than one per site, the chemical potential is located at the top of the Hubbard lower band of the insulator. We consider the reconstruction of FBZ boundary leading to the folded Fermi surface instability at phase separation. In spite of an absence of long-range $\mathrm{AF}$ in our calculation, the local AF order in inhomogeneous system still can occur. The above conclusion can be used to explain the lack of the phase separation in the honeycomb lattice. By comparing with the results of isolated clusters, we can identify the spectrum around $( \pm \pi / 2 a, \pm \pi / 2 a)$ points which are playing the same role as the $N-1$ electron state in an isolated clusters [7]. Although a local AF state in the honeycomb lattice exists for all $U$ as in the square lattice, phase separation that is seen in the square lattice does not occur in the honeycomb lattice. The smooth transition in honeycomb lattice is also confirmed in quantum cluster calculations for ensemble of independent hexagon rings [7]. The reason is that no matter whether the honeycomb lattice has (basic) AF order or not, the size of the primitive unit cell does not change (two sites per primitive cell). When the system undergoes a phase transition from the local AF state to the normal state, the lattice parameter does not change, i.e., no change in the AF FBZ boundaries. One-particle spectral function shows smooth evolution in the vicinity of the Fermi level without sign of spectrum anomaly near Fermi. This implies the absence of the stable mixed PS phase in hexagonal structure, since there is no FBZ-folding.

\section{Summary}

The VCA tool without long range order input allows treat both the metallic and the Mott insulating phase at the close vicinity of the quantum 
critical point on equal footing. We studied phase separation in bipartite square and honeycomb lattices in the Hubbard model by looking for different electronic configurations with different electron density at the same chemical potential for thermodynamic stability. The obtained smooth Mott-Hubbard metal-insulator transition at half filling agrees with continuous transition at finite $U / t$ value in [34]. In addition, our VCA results show the discontinuity and phase separation in the presence of a finite carrier density $(n \neq 1)$ [35]. The first-order magnetic transitions can also influence a sharp variation in terms of electron density at the vicinity of half-filling. We observe a phase separation in form of a discontinuity in the density in the weakly doped square lattice model correlated with the changes in the single-particle spectrum near the $\mathrm{Q}$ point $( \pm \pi / 2 a, \pm \pi / 2 a)$. Our VCA studies of the honeycomb lattice, i.e., graphene layer, show no sign of phase separation, i.e., the lattice geometry plays a crucial role in folding or unfolding AF FBZ. In the square lattice, a small part of spectrum, which is tied to the Fermi surface instability at the FBZ boundary, disappears when Mott insulating phase destroys the electron-hole symmetry by doping. The honeycomb lattice has a different lattice geometry, which leaves the FBZ unfolded under doping, so the honeycomb lattice does not show phase separation instability and spatial inhomogeneities in entire $U$ region. In our VCA calculations without input of long-range order parameter, we observe a phase separation at moderate $U>U_{C}$ between an insulator and a metal, while in the dynamical mean field theory (DMFT) the phase separation occurs between two different metallic phases [11]. The obtained here scenario of phase separation under doping at finite $U>U_{\mathrm{C}}$ is also different from the jump like electronic transitions using the mean field order parameter in the two orbital model [27]. The obtained discontinuity in electron (charge) density at $U>U \mathrm{c}$ in square Hubbard lattice away from half filling closely resembles the first order electron phase transitions with coexistence of two different electron configurations at the same chemical potential. It is noteworthy to say that phase separation seen in transition-metal oxides and superconducting cuprates at relatively weak electron correlations is different from magnetic phase separation instabilities in the pyrochlores, and other magnetic materials driven by spin density transitions at large $U$. The VCA picture of phase transition discontinuity at weak and moderate $U$ is consistent with our quantum cluster calculations. The ground state results provide insight into possible mechanism of spontaneous phase separation under change of carrier concentration and $U$ (parameterized by pressure) in square lattice structures. Our VCA provides substantial support for phase-separation instability obtained earlier at the quantum cluster level [36-40], i.e., phase separation in square clusters and its absence in honeycomb cluster geometry persists also in larger size lattices. The obtained picture of phase separation is consistent with the STM observations of nanoscale inhomogeneities dominated by superconducting hole-rich and the local AFM holepoor regions coexisting without an apparent longrange order [41-42].

\section{Acknowledgements}

The authors would like to thank Jian-Xin Zhu and acknowledge the computing facilities provided by the Center for Integrated Nanotechnologies, the U.S. Department of Energy, Office of Basic Energy Sciences user facility at Los Alamos National Laboratory (Contract DE-AC52-06NA25396). The work was performed also, in part, at the Center for Functional Nanomaterials, Brookhaven National Laboratory supported by the U.S. Department of Energy, Office of Basic Energy Sciences, under the Contract No.DE-AC02-98CH10886.

\section{References}

[1] J.M. Tranquada, B.J. Sternlieb, J.D. Axe, Y. Nakamura, S. Uchida, Nature (London) 375 (1995) 561; E. Dagotto, Rev. Mod. Phys. 66 (1994) 763; E. L. Nagaev, Phys. Usp. 38 (1995) 497.

[2] J.E. Hoffman, E.W. Hudson, K.M. Lang, V. Madhavan, H. Eisaki, S. Uchida, J.C. Davis, Science 295 (2002) 466.

[3] P.B. Visscher, Phys. Rev. B 10 (1974) 943.

[4] V.J. Emery, S.A. Kivelson, H.Q. Lin, Phys. Rev. Lett. 64 (1990) 475.

[5] V. Emery, S. Kivelson, Physica C: Superconductivity 209 (1993) 597.

[6] A.N. Kocharian, G.W. Fernando, K. Palandage, J.W. Davenport, J. Magn. Magn. Mater., 300 (2006) e585.

[7] A.N. Kocharian, G.W. Fernando, K. Palandage, J.W. Davenport, Phys. Rev. B74 (2006) 024511.

[8] W.-F. Tsai and S.A. Kivelson, Phys. Rev. B73, 214510, 2006 [9] A.N. Kocharian, Kun Fang, G.W. Fernando, J. Magn. Magn. Mater. 324 (2012) 3427.

[10] M. Aichhorn, E. Arrigoni, M. Potthoff, W. Hanke, Phys. Rev. B 74 (2006) 235117; ibid B 76 (2007) 224509.

[11] R.S. Markiewicz, J. Phys.: Condens. Matter 2 (1990) 665. 
[12] G. Sordi, K. Haule, A.-M.S. Tremblay, Phys. Rev. B84 (2011) 075161

[13] G. Sordi, K. Haule, A.-M.S. Tremblay, Phys. Rev. Lett. 104 (2010) 226402

[14] M. Capone, G. Sangiovanni, C. Castellani, C.Di Castro, M. Grilli, Phys. Rev. Lett. 92 (2004) 106401.

[15] B.V. Fine, T. Egami, Phys. Rev. B77 (2008) 014519.

[16] M. Jarrell, Phys. Rev. Lett. 69 (1992) 168.

[17] A. Georges, G. Kotliar, W. Krauth, M.J. Rozenberg, Rev. Mod. Phys. 68 (1996) 13.

[18] M. Potthoff, M. Aichhorn, C. Dahnken, Phys. Rev. Lett. 91 (2003) 206402.

[19] T. Maier, M. Jarrell, T. Pruschke, M.H. Hettler, Rev. Mod. Phys. 77 (2005) 1027.

[20] M. Potthoff, The European Physical Journal B: Condensed Matter and Complex Systems 32 (2003) 429

[21] M. Potthoff, M. Aichhorn, C. Dahnken, Phys. Rev. Lett. 91 (2003) 206402

[22] D. S’en'echal, D. Perez, M. Pioro-Ladri`ere, Phys. Rev. Lett. 84 (2000) 522

[23] J. Cullum and R. Willoughby, Lanczos Algorithms for Large Symmetric Eigenvalue Computa-tions: V.1, Theory, Classics in Applied Mathematics (Society for Industrial and Applied Mathematics, 2002).

[24] Y.Z. Zhang, M. Imada, Phys. Rev. B76 (2007) 045108.

[25] S. Sorella, E. Tosatti, Europhys.Lett. 19 (1992) 699.

[26] A. Macridin, M. Jarrell, T. Maier, Phys. Rev. B74 (2006) 085104 .

[27] A.N. Kocharian, D.I. Khomskii, Solid State Commun. 18 (1976) 985; Sov. Phys. Solid State, 14 (1973) 2093.

[28] M. Calamiotou, A. Gantis, D. Lampakis, E. Siranidi, E. Liarokapis, I. Margiolaki, K. Conder, Europhys. Lett. 85 (2009) 26004.

[29] E. Liarokapis, D. Lampakis, E. Siranidi, and M. Calaniotou, J. Phys. Chem. Sol., 71 (2010) 1084.

[30] D. Lampakis, In: Bianconi ed., Symmetry and Heterogeneity in High Temperature Superconductors, NATO Science Series II Mathematics,Physics and Chemistry. Phase separation in cuprates induced by doping, hydrostatic pressure or atomic substitution. V. 214 A (2006) 117-132 Springer, Dordrecht, The Netherlands.

[31] N. Furukawa, T.M. Rice, M. Salmhofer, Phys. Rev. Lett. 81 (1998) 3195 .

[32] N. Furukawa, T.M. Rice, J. Phys.: Condens. Matter 10 (1998) L381.

[33] A.O. Sboychakov, A.V. Rozhkov, K.I. Kugel, A.L. Rakhmanov, Franco Nori, B 88 (2013) 195142; B87 (2013) 075128.

[34] M. A. Timirgazin, A. K. Arzhnikov, and V. Yu. Irkhin, JETP Letters, 96 (2012) 171-175.

[35] P. A. Igoshev, M. A. Timirgazin, A. A. Katanin, A. K. Arzhnikov and V. Yu. Irkhin, Phys. Rev. B81 (2010) 094407

[36] G.W. Fernando, A.N. Kocharian, K. Palandage, T. Wang, J.W. Davenport, Phys. Rev. B75 (2007) 085109.

[37] K. Palandage, G.W. Fernando, A.N. Kocharian, J.W. Davenport, J. Comput. Aided Mater. Des. 14 (2007) 103

[38] A.N. Kocharian, G.W. Fernando, K. Palandage, J.W. Davenport, Phys. Rev. B78 (2008) 075431.

[39] G.W. Fernando, K. Palandage, A.N. Kocharian, J.W. Davenport, Phys. Rev. B80 (2009) 014525.

[40] A.N. Kocharian, G.W. Fernando, K. Palandage, J.W. Davenport, Phys. Lett. Phys. Lett. A373 (2009) 1074; ibid A364 (2007) 57.
[41] T. Egami, In: Bussmann-Holder A, Keller H (eds)

High Tc Superconductors and Related Transition Metal Oxides. Springer-Verlag Berlin Heidelberg, 103-129 (2007);

[42] A. Damascelli, D.H. Lu, Z.-X. Shen, J. Electron Spectros. Related Phenomena 117-118 (2001) 165. 